\title{
Incidental Detections Suggestive of COVID-19 in Asymptomatic Patients Undergoing 68Ga-DOTATATE and 68Ga-PSMA-11 PET-CT Scan for Oncological Indications
}

\section{COVID-19 Zufallsbefund bei asymptomatischen Patienten mit 68Ga-DOTATATE und 68Ga-PSMA-11 PET-CT-Untersuchung aufgrund onkologischer Indikation}

\section{Introduction}

A novel severe acute respiratory syndrome coronavirus 2, SARS-CoV-2, has emerged in China in December 2019, resulting in an atypical pneumonia called Corona Virus Disease 2019 (COVID-19). The disease has continued to extend globally as the first $21^{\text {st }}$ century pandemic [1]. Despite the suboptimal sensitivity of polymerase chain reaction (RT-PCR) assay, it has been widely considered as the gold standard of diagnosis in the early stages of SARS-CoV-2 infection. Chest computed tomography has been employed in many countries as a substitute or complementary tool for screening/diagnosis, assessment of severity of the disease, and monitoring of response to treatment of COVID-19 pneumonia. It provides higher sensitivity compared to RT-PCR (98\% versus $71 \%$ ). In many patients, COVID-19 may present with non-specific respirator and/or constitutional symptoms, such as fever, cough, and dyspnea, while in some others, may remain completely asymptomatic [2-7].

Recently, it has been a growing number of publications about the role of 18F-FDG PET scan as a non-invasive tool [8, 9]. The importance of these incidental findings is mainly because these patients constitute a more susceptible population to the disease complications (such as ICU admission, need for intubation, and death) due to their underlying conditions, such as cancer $[10,11]$. In these cases, PET/CT may show increased FDG uptake in mediastinal lymph nodes and mild uptake in lung parenchyma with observation of multilobar multifocal ground-glass opacities in the CT portion of the exam. In an acute chest infection, such as COVID-19, inflammatory cells are activated, resulting in release of local inflammatory chemokines. Accordingly, activated neutrophils, which depend on glycolysis, infiltrate into the lung parenchyma, which manifest as high FDG uptake on PET/CT. Previous studies confirmed that increased FDG uptake in
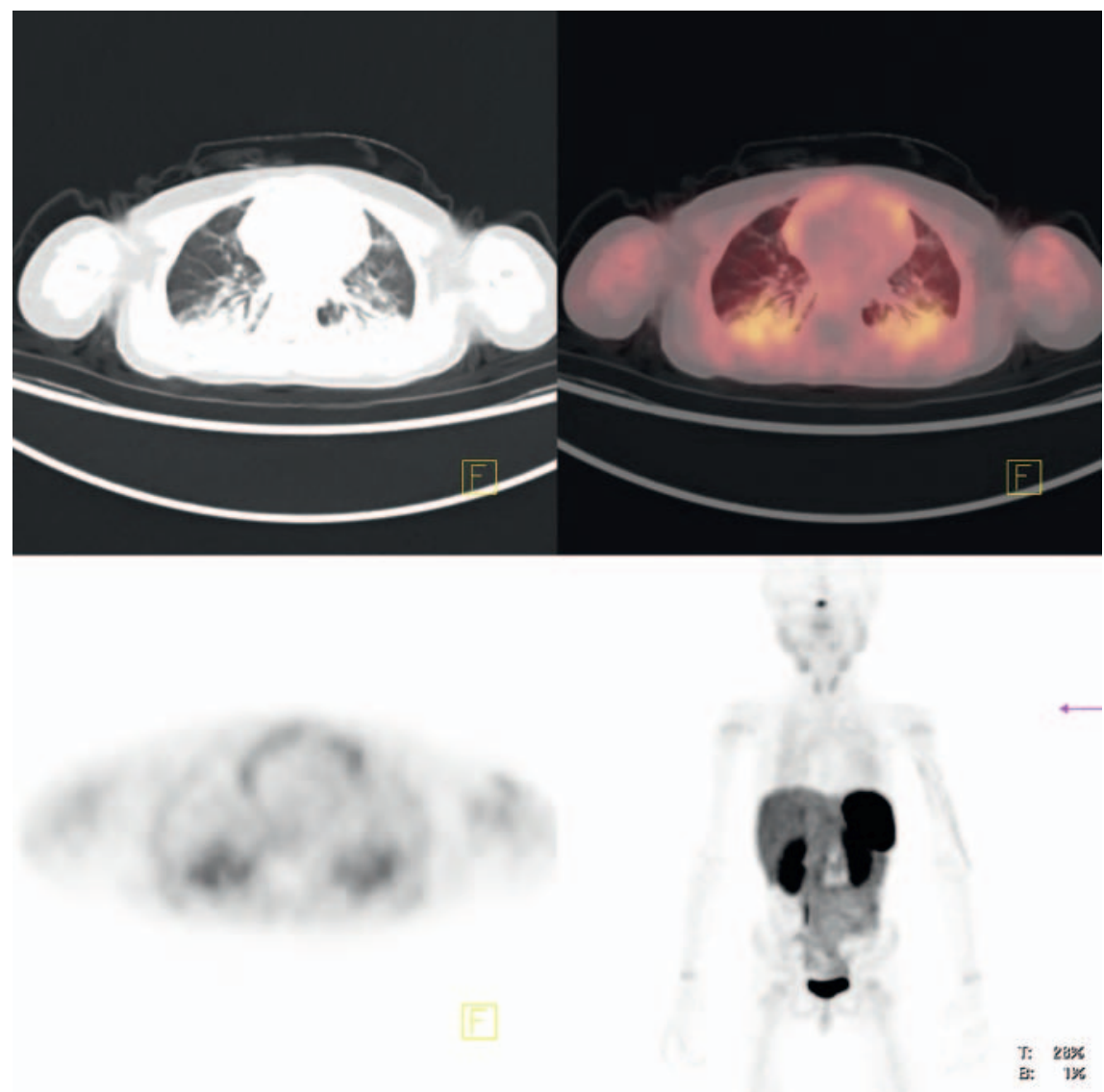

- Fig. 1 68Ga-DOTATAE PET/CT scan was performed, which demonstrated bilateral peripheral dependent ground glass opacities with superimposed consolidations. Increased radiotracer uptake (SUVmax $=2.45$ ) was also noted, highly suspicious for COVID-19.

mediastinal and axillary lymph nodes is associated with increased monocytes infiltration as the main response of lymphoid tissue to scrim viral infections [12]. Several groups reported incidental finding of COVID-19 in asymptomatic patients undergoing 18F-FDG PET/CT and 131 I SPECT/CT scans for oncological indications $[2,8,13]$. Based on these observations, nuclear medicine physician and nuclear radiologists should be familiar with the imaging features of the disease. Understanding the imaging findings of COVID19 and other pulmonary infections is crucial to ensure appropriate and timely diagnosis.
Here, we report 68Ga-DOTATATE and 68Ga-PSMA-11 PET-CT imaging findings of two asymptomatic coronavirus infection patients who were admitted to our nuclear medicine department for oncological indications, resulting in incidental identification of previously unsuspected COVID-19.

\section{Case Report}

\section{Case 1}

A four-year-old girl with severe hypoglycemia presented to our nuclear medicine department for 68Ga-DOTATATE PET/CT to 
exclude possible pancreatic neuroendocrine tumor. Firstly, she underwent abdominal-pelvic CT scan which was negative for any abnormality. Laboratory testing revealed a white blood cell (WBC) count within the reference range $(6.31000 / \mu \mathrm{L})$ and also a normal range of serum Creatinine $(0.7 \mathrm{mg} / \mathrm{dL}$, reference range 0.6 $1.3 \mathrm{mg} / \mathrm{dL}$ ). Intravenous dose of $51.8 \mathrm{MBq}$ (1.4 mCi) 68Ga-DOTATATE was injected and after 1 hour the 68Ga-DOTATATE PET/ CT scan was performed using an integrated 6-slice PET/CT scanner. The study revealed no nodal uptake in the mediastinum, but multiple peripheral ground-glass opacities (SUVmax $=2.45$ ) were identified, highly concerning for COVID-19 ( $\triangleright$ Fig. 1). RTPCR for COVID-19 was positive as well as the serology test detected SARS-CoV-2 IgG and SARS-CoV-2 IgM were positive (13.5 and $1.7 \mathrm{IU} / \mathrm{mL}$ respectively).

\section{Case 2}

A 81-year-old man with relapsed prostate cancer, status post androgen deprivation therapy (ADT), presented to our nuclear medicine department. Laboratory testing showed PSA $=240 \mathrm{ng} / \mathrm{mL}$ and white blood cell (WBC) count within the reference range (10.10 1000/ $\mathrm{LL}$ ) and a low elevation of Creatinine $(1.8 \mathrm{mg} / \mathrm{dL}$, reference range $0.6-1.3 \mathrm{mg} / \mathrm{dL}$ ). Additionally, the number of platelets was decreased to $1251000 / \mu \mathrm{L}$ (reference range 140-450 1000/ $\mu \mathrm{L}$ ). A high-resolution chest computed tomography (HR-CT) was previously performed, which revealed a soft tissue density non-calcified right peripheral pulmonary nodule with irregular borders $(9 \mathrm{~mm}$ in diameter). Scattered smaller soft tissue density pulmonary nodules of 3 to $4 \mathrm{~mm}$ in diameter were also identified involving bilateral lungs. The bone scintigraphy was negative for metastatic osseous disease. At our institution, an intravenous dose of 181.3 MBq (4.9 mCi) 68Ga-PSMA-11 was injected. After 1 hour, imaging was performed on an integrated 6-slice PET/CT scanner, with scanning from the skull top to the mid-thigh.

The 68Ga-PSMA-11 PET/CT scan revealed a right supraclavicular lymph node with increased PSMA uptake (SUVmax=8.91). Multiple mediastinal lymph nodes were noticed in the right upper paratracheal, subcarinal, retroesophageal, and left para-

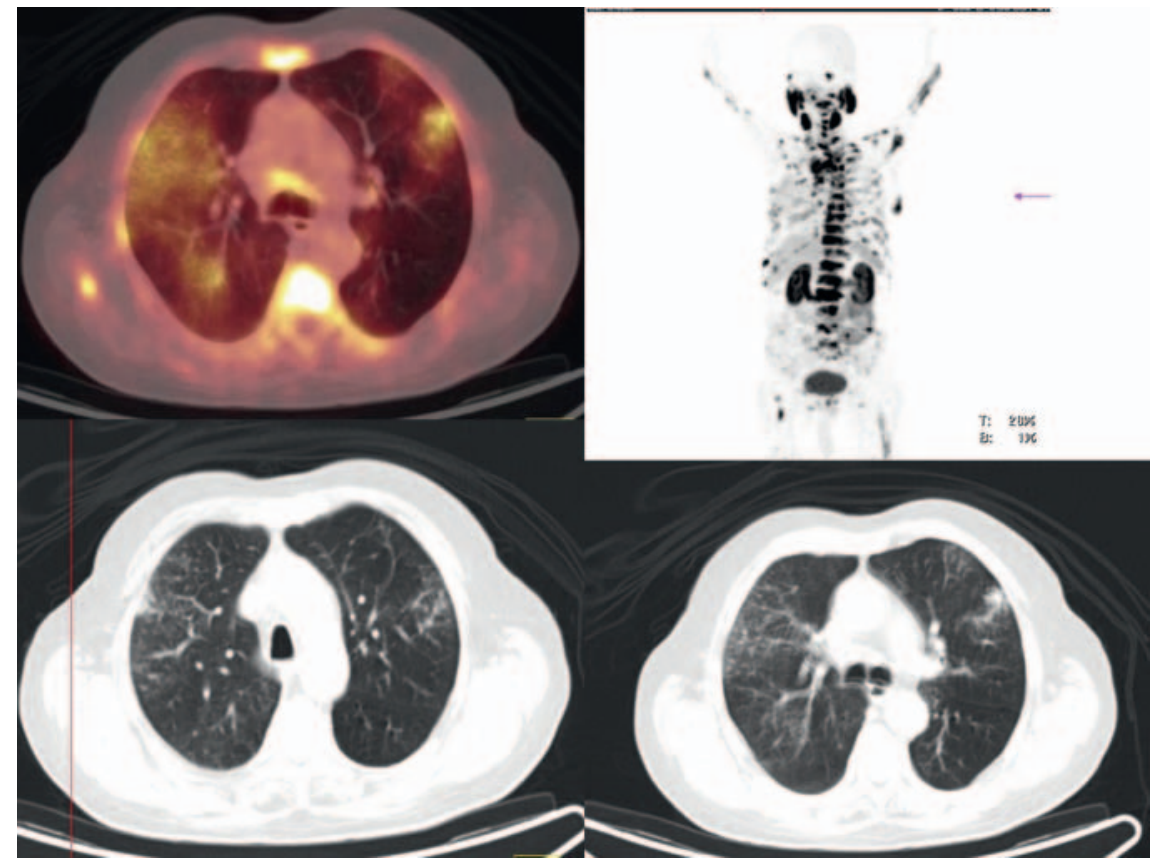

- Fig. 2 68Ga-PSMA-11 scan performed for an 81-year-old man suspected to have pulmonary metastases of prostate cancer. The 68Ga-PSMA-11 PET/CT scan revealed bilateral ground-glass opacities and consolidations with mildly increased PSMA uptake, concerning for COVID-19.

tracheal compartments. A soft tissue density non-calcified right upper lobe pulmonary nodule with increased tracer uptake (SUVmax = 5.42), left upper lobe pulmonary nodule (posterior segment) with increased tracer uptake (SUVmax $=4.17$ ), as well as another small right hilar pulmonary nodule (SUVmax $=6.53$ ) were identified. Also interlobular septal thickening showing increased activity (SUVmax $=6.53$ ). Incidentally, bilateral ground-glass opacities were noted involving bilateral lungs with mildly increased PSMA uptake, highly concerning for COVID-19. However, initial SARS-CoV-2 RT-PCR was negative ( $\triangleright$ Fig. 2 ).

\section{Discussion}

There have been several reports in recent literature regarding incidental detection of COVID-19 on 18F-FDG PET/CT scans. Although no role for diagnosis of SARSCoV-2 infection by PET/CT can be considered, the incidental finding of the disease on PET/CT imaging can have a significant effect on the patient management and should be reported carefully. In fact, the interpretation of PET/CT studies should not be limited to the evaluation of oncologic findings. With the increasing number of the SARS-CoV-2 infection worldwide, interpreting radiologist and nuclear medicine physicians should be completely trained and aware of the imaging features of COVID-19 pneumonia. More specifically, constant vigilance is required to include COVID-19 in the differential diagnosis in patients with suspicious imaging features. PET has shed light particularly at early and non-specific stages of the disease with challenging clinical symptoms [8-11, 13, 14].

In our case report, we described 68GaDOTATATE and 68Ga-PSMA-11 imaging of two patients with asymptomatic COVID-19. The role of these two radiotracers in the diagnosis, post-treatment follow-up, and management of neuroendocrine and prostate malignancies is well known. We strongly recommend the evaluation of PET/CT images for non-oncologic conditions in daily practice of radiology and nuclear medicine, as this may lead to timely detection of crucial non-oncologic pathologies of high clinical importance in patient management. These days, extremely high clinical index of suspicion for COVID-19 and low threshold for paraclinical screening and diagnostic testing of SARS-CoV-2 infection, such as PCR and CT chest, allows earlier diagnosis and treatment of this potentially life-threatening condition. Timely detection of the 
disease by the interpreting radiologist also can help ensure appropriate post-exposure precautions in nuclear medicine departments are implemented and the staff and other patients are protected.

Conflict of Interest

The authors declare that they have no conflict of interest.

\section{Authors}

Habibollah Dadgar ${ }^{1}$, Nasim Norouzbeigi ${ }^{1}$, Ali Gholamrezanezhad ${ }^{2}$, Majid Assadi ${ }^{3}$

${ }^{1}$ The Cancer Research Center, Razavi Hospital, Imam Reza International University, Mashhad, Iran

${ }^{2}$ Department of Radiology, Keck School of Medicine, University of Southern California (USC), 1500 San Pablo Street, Los Angeles, CA 90033 USA

${ }^{3}$ The Persian Gulf Nuclear Medicine Research Center, Department of Molecular Imaging and Radionuclide Therapy, Bushehr Medical University Hospital, Bushehr University of Medical Sciences, Bushehr, Iran

\section{Correspondence}

\section{Prof. Majid Assadi}

The Persian Gulf Nuclear Medicine Research Center, Department of Molecular Imaging and Radionuclide Therapy, Bushehr Medical University Hospital, Bushehr University of Medical Sciences Bushehr

Iran

assadipoya@yahoo.com

\section{Reference}

[1] Lütje S, Marinova M, Kütting D et al. Nuclear medicine in SARS-CoV-2 pandemia: 18F-FDG$\mathrm{PET} / \mathrm{CT}$ to visualize COVID-19. Nuklearmedizin 2020; 59 (3): 276-280. English. doi:10.1055/a-1152-2341. Epub 2020 Apr 7. PMID: 32259853

[2] Albano D, Bertagna F, Bertoli M et al. Incidental Findings Suggestive of COVID-19 in Asymptomatic Patients Undergoing Nuclear Medicine Procedures in a High-Prevalence Region. J Nucl Med 2020; 61 (5): 632-636. doi:10.2967/jnumed.120.246256. Epub 2020 Apr 1. PMID: 32238429

[3] Huang C, Wang Y, Li X et al. Clinical features of patients infected with 2019 novel coronavirus in Wuhan, China. Lancet 2020; 395 (10223): 497506. doi:10.1016/S0140-6736(20)30183-5. Epub 2020 Jan 24. Erratum in: Lancet. 2020 Jan 30. PMID: 31986264 ; PMCID: PMC7159299

[4] Shi H, Han X, jiang $N$ et al. Radiological findings from 81 patients with COVID-19 pneumonia in Wuhan, China: a descriptive study. Lancet Infect Dis 2020; 20 (4): 425-434. doi:10.1016/S1473-3099(20)30086-4. Epub 2020 Feb 24. PMID: 32105637 ; PMCID: PMC7159053

[5] Li G, Li W, He X et al. Asymptomatic and Presymptomatic Infectors: Hidden Sources of Coronavirus Disease 2019 (COVID-19). Clin Infect Dis 2020; 71 (8): 2018. doi:10.1093/cid/ciaa418. PMID: 32271372; PMCID: PMC7184467

[6] Assadi M, Gholamrezanezhad A, Jokar N et al. Key elements of preparedness for pandemic coronavirus disease 2019 (COVID-19) in nuclear medicine units. Eur J Nucl Med Mol Imaging 2020; 47 (8): 1779-1786. doi:10.1007/s00259-020-04780-4. PMID: 32318782; PMCID: PMC7171056

[7] Salehi S, Abedi A, Balakrishnan S et al. Coronavirus disease 2019 (COVID-19) imaging reporting and data system (COVID-RADS) and common lexicon: a proposal based on the imaging data of 37 studies. Eur Radiol 2020; 30 (9): 4930-4942. doi:10.1007/s00330020-06863-0. Epub 2020 Apr 28. PMID: 32346790; PMCID: PMC7186323

[8] Qin C, Liu F, Yen TC et al. 18F-FDG PET/CT findings of COVID-19: a series of four highly suspected cases. Eur J Nucl Med Mol Imaging 2020; 47 (5): 1281-1286. doi:10.1007| s00259-020-04734-w. Epub 2020 Feb 22 PMID: 32088847; PMCID: PMC7080035
[9] Deng Y, Lei L, Chen Y et al. The potential added value of FDG PET/CT for COVID-19 pneumonia. Eur J Nucl Med Mol Imaging 2020; 47 (7): 1634-1635. doi:10.1007| s00259-020-04767-1. Epub 2020 Mar 21. PMID: 32198615; PMCID: PMC7087529

[10] Alberts I, Vollnberg B, Sachpekidis C et al. Incidental SARS-CoV-2-related findings in asymptomatic patients in [18F]-FDG-PET/ CT-potential insights. Eur ] Nucl Med Mol Imaging 2020; 47 (9): 2068-2069. doi:10.1007/s00259-020-04869-w. Epub 2020 May 22. PMID: 32444898; PMCID: PMC7244259

[11] Kooraki S, Hosseiny M, Myers L et al. Coronavirus (COVID-19) Outbreak: What the Department of Radiology Should Know. J Am Coll Radiol 2020; 17 (4): 447-451. doi:10.1016/j.jacr.2020.02.008. Epub 2020 Feb 19. PMID: 32092296; PMCID: PMC7102595

[12] Chefer S, Thomasson D, Seidel J et al. Modeling [(18)F]-FDG lymphoid tissue kinetics to characterize nonhuman primate immune response to Middle East respiratory syndrome-coronavirus aerosol challenge. EJNMMI Res 2015; 5 (1): 65. doi:10.1186/ s13550-015-0143-x. Epub 2015 Nov 16. PMID: 26573211; PMCID: PMC4646887

[13] Martineau P, Kidane B. FDG PET/CT Findings in an Asymptomatic Case of Confirmed COVID-19. Clin Nucl Med 2020; 45 (8): 647648. doi:10.1097/RLU.0000000000003145. PMID: 32433160; PMCID: PMC7268853

[14] Playe M, Siavellis ], Braun T et al. FDG PET/CT in a Patient With Mantle Cell Lymphoma and COVID-19: Typical Findings. Clin Nucl Med 2020; 45 (7): e305-e306. doi:10.1097| RLU.0000000000003113. PMID: 32453077; PMCID: PMC7268867

\section{Bibliography}

Nuklearmedizin 2021; 60: 106-108 Published online: December 16, 2020 DOI 10.1055/a-1311-2856

ISSN 0029-5566

(C) 2020. Thieme. All rights reserved.

Georg Thieme Verlag KG, Rüdigerstraße 14, 70469 Stuttgart, Germany 